\title{
La "bella scola" ed il "salutevol cenno": una ricerca semantica e stilistica
}

Quando Dante è accolto nella "bella scola"1 del Limbo (Inf. 4.80-102) la sua formale 'accettazione' nel mezzo dei massimi poeti del canone da lui stesso creato avviene per mezzo di un generale "salutevol cenno" seguito poi da un compiaciuto sorriso virgiliano. I quattro grandi poeti del canone "ragionano" di Dante piuttosto a lungo prima di ammetterlo fra di loro e tutta l'atmosfera dell'episodio è fortemente connotata nel senso di una 'investitura' cavalleresco-accademica.

Verso una dimensione 'cortese' dell'incontro ci spinge soprattutto la descrizione di Omero, che è "poeta sovrano" e che, brandendo una spada, incede "come sire". Il massimo poeta della tradizione occidentale è poi paragonato all'aquila, animale 'imperiale' per eccellenza (due sole occorrenze nel poema: qui e nell'episodio di Giustiniano di Par. 6.1) e Dante è reso membro di una "schiera", vocabolo che curiosamente compare nella sua piena valenza guerresca (valenza che nel poema è assunta dal vocabolo solo nel caso seguente su 19 occorrenze) nell'episodio di Forese Donati (Purg. 24.99) in cui Virgilio e Stazio, poeti anch'essi, son per giunta definiti "marescalchi" (unica occorrenza nel poema).

Altro indizio "cortese' è il verbo-tecnico "ragionare" ("Amor che nella mente mi ragiona") che - pertinente ad un piano epidittico o dimostrativo, come ben si vede dallo studio dell'uso che ne fa Dante nel caso del 'conciliabolo' che stiamo esaminando - viene a mio giudizio ricondotto alla sua specializzazione curtense o lato sensu stilnovistica di 'amabile dialogo di nobili intelletti' come in Purg. 2.112; Purg. 22.21, 104 e 130 (nel canto di Stazio); Purg. 24.2 (proprio nel canto di Forese) e nell'importantissimo commiato di Virgilio in Purg. 27.53.

Il vocabolo "senno"2 ricorre 11 volte nel poema in chiusura di verso (ma globalmente 15) ed in 9 di queste occasioni fa rima con "cenno". Non credo che si tratti soltanto di una costrizione del rimario dantesco, che effettivamente come forme concorrenziali offre solo il toponimo "Lenno" o le tre forme verbali "enno", "dienno" e "fenno", ma penso che si possa parlare piuttosto di un particolare rilievo dato al rapporto allievo-maestro, nell'estrinsecarsi del quale ad una positiva valutazione dell'ingegno non può non far seguito un "cenno" di 
approvazione se non proprio una lode. ${ }^{3}$ E questo da un funto di vista 'accademico' è pacifico; ma il principio vale, e forse ancora di più, da un punto di vista psicagogico o comunque esemplare, se viene applicato a persone che condividono un particolare status, una condizione privilegiata di appartenenza ad una élite culturale o, meglio, spirituale, capace di incarnare ideali tali da poter giustificare la 'solennità' ed allo stesso tempo la 'complicità' di questo tipo di 'saluto-segnale', di questo 'riconoscimento' tra pari o comunque fra persone che, se non esattamente pari per dignità, appartengono ad un mondo 'superiore" di cui l' 'annuizione' è segno tangibile (visto che d'altra parte adnuere, cioè 'fare un cenno di approvazione', era il verbo tecnico usato dai latini per indicare il 'favore' della divinità nei confronti di un difficile progetto o di un 'ardua impresa, e specialmente nell'epica). Per quanto riguarda "cenno" il Thesaurus Linguae Latinae, pur parlando di origo incerta, conferma per il lemma cinnus una parentela con l'atto dell'annuizione, se nelle Glosse néuma è equiparato a nutus e se in Fulgenzio (Serm. ant.46) si attesta: nictare dicimus cinnum facere. Il Dizionario Etimologico Italiano, dopo aver menzionato le stesse fonti del Thesaurus, si spinge più in là citando quelle che vengono definite le "contaminazioni" del vocabolo cinnum con signum che sarebbero avvenute nei dialetti italiani del nord (genovese, piemontese, emiliano) ed addirittura avanzando l'ipotesi etimologica di una connessione fra il latino cinnum ed il greco kinéo (= 'muovo', che instaura, dal punto di vista della analisi che ci accingiamo a presentare, una suggestiva relazione causa-effetto fra 'cenno' e 'movimento', o 'avanzamento'). Molto laconica è invece la voce "cenno" del Dizionario Etimologico della Lingua Italiana di Cortellazzo-Zolli, laddove assai doviziosa di riscontri è la stessa voce nel Grande Dizionario della Lingua Italiana curato dal Battaglia; riscontri che però non aggiungono molto all'idea generale di "ordine". "comando" o "segno", "segnalazione". Per quello che riguarda invece il vocabolo "senno", oltre alla solita laconicità del DELI, registriamo una se non esauriente quanto meno sufficiente trattazione nel $D E I$ che avanza l'etimologia dal francone $\sin$ o sinn che varrebbe "senno", "tendenza", significato che permane nel tedesco sinn che significa appunto, secondo il Wahrig, 'senso', 'organo di senso', 'sensibilita', 'comprensione', 'scopo' e 'significato’. La lettura del $G D L I$ è invece per noi assai importante perché fra i numerosissimi riscontri del termine che vengono proposti, e che ovviamente comprendono i significati più prevedibili di "prudenza", "buon senso", "facoltà intellettiva", "disposizione spirituale" e "astuzia", c’è anche una attestazione del vocabolo usato nel senso di "criterio" o "principio informatore" (Ariosto, Furioso 4.65). Come è chiaro una citazione da Ariosto sarebbe irrilevante ai fini dello studio che ci proponiamo se non avesse appigli anche nel corpus dantesco. E assai stranamente i compilatori della voce hanno relegato una interessantissima occorrenza del termine nel Convivio (4.27.9), perfettamente sovrapponibile a quella dell 'Ariosto, nella categoria "buon senso". Crediamo infatti che nel luogo dantesco "senno" valga "conoscenza e possesso di 
un codice morale'; acquisizione che in certo modo ci agevolerà nell 'affrontare il nostro viaggio attraverso la strategia intratestuale di Dante che abbiamo posto a oggetto della nostra ricerca. Utili ma non dirimenti e soprattutto non apportatrici di considerazioni nuove sono poi le voci cenno e senno dell 'Enciclopedia Dantesca, compilate rispettivamente da Emilio Pasquini e Alessandro Niccoli. Vediamo di ripercorrere, a questo punto, lo sviluppo all'interno del poema e, per così dire, la 'casistica' di questi complici riconoscimenti o 'investiture'.

Dobbiamo partire evidentemente con il canto 4 dell'Inferno che non riesamineremo nel suo complesso ma di cui mi piace far notare qualche aspetto rimasto fin qui in ombra. Dato che ogni vera 'annuizione' deve avere una funzione diciamo così propiziatrice (si noti l'ambiguo valore semantico di "cenno salutevol" a mezza via fra 'cenno di saluto' e "cenno di buon auspicio" o 'cenno che conforta e rinfranca' visto il significato del latino salus), l'ingresso di Dante in una "compagnia" proprio al principio di un viaggio, per quanto breve sia il tratto di cammino che il pellegrino compie con i cinque personaggi, può avere secondo me la funzione di un potente "viatico' ( del resto anche il valore semantico di "compagnia" come "gruppo di persone con cui condividere delle difficoltà', delle prove o dei disagi è ben attestato nella Commedia).

Nella seconda delle occorrenze (Inf. 8.3-9) del gruppo "cenno-senno", e cioè nella descrizione della sosta di Dante e Virgilio di fronte alle mura della città di Dite, l'enfasi si pone in senso passivo proprio sulla oscurità di un "cenno" (cioè di un segnale convenuto, un segno di riconoscimento) di cui Dante non conosce il 'codice', cosicché lo smarrito pellegrino interroga Virgilio, dopo averlo qualificato "mar di tutto il senno", proprio sulla natura delle segnalazioni luminose che si scambiano dalle torri della città.

[...]li occhi nostri n'andar suso a la cima
per due fiammette che i vedemmo porre,
e un'altra da lungi render cenno,
tanto ch a pena il potea l'occhio tòrre.
E io mi volsi al mar di tutto 'l senno;
dissi : "Questo che dice? e che risponde
quell'altro foco? e chi son quei che 'l fenno?"

Questo senso di disvelamento di un arcano pervade poi profondamente il terzo episodio, quello dell' ascesa di Gerione sollecitata da Virgilio per mezzo della corda che Dante usava come cintola (Inf. 16.115-20). Virgilio riceve da Dante la corda e la getta nel burrone; a questo punto il poeta si chiede quale novità debba seguire a questo nuovo "cenno" (ciod inequivocabilmente un "segnale" come tutti i più autorevoli commentatori concordano nell 'affermare) fatto dal suo maestro a chissà quale entità. Virgilio che è il "mare" di ogni possibile ingegno non soltanto è pienamente consapevole del suo proprio gesto, 
ma addirittura 'legge' il pensiero di Dante sciogliendo i suoi dubbi e fornendogli in anticipo una risposta. Il poeta commenta questa dimostrazione d'acume da parte di Virgilio ammonendo le persone comuni a non tentare di dissimulare le cause delle proprie azioni di fronte ad uomini di ingegno tale che possano 'leggere il pensiero' ("ma per entro i pensier miran col senno"):
"E' pur convien che novità risponda", dicea fra me medesmo, "al novo cenno che 'l maestro con l'occhio sì seconda". Ahi quanto cauti li uomini esser dienno presso a color che non veggion pur l'ovra, ma per entro i pensier miran col senno!

Il primo dei due canti dedicati alla Bolgia dei Barattieri si conclude, come è noto, (Inf. 21.131-39) con l'inganno di Malacoda che affida Dante e Virgilio ad una scorta di diavoli perché siano condotti alla sesta Bolgia. Anche in questo caso l'uso del vocabolo "cenno" è da ricondurre esattamente al campo semantico di 'segnale', 'segno convenzionale' e nell'economia dell'espressionismo stilistico tipico del canto esso si riferisce al fatto che, nel congedarsi e nel voltare verso l'argine sinistro della scarpata, ogni diavolo stringe la lingua fra $i$ denti rivolgendosi al capo della schiera ("verso lor duca") proprio come a indicare il tipo di segnale che si aspettano ("per cenno"), ed il capo dà appunto il volgare incipit della sua famosa flatulenza ("ed elli avea del cul fatto trombetta") affinché essi possano rispondere con le loro pernacchie. Virgilio, a cui Dante aveva manifestato timore per il fatto che i diavoli "minaccian duoli" col digrignare i denti, aveva già ammonito il suo discepolo in merito ai "costumi' diabolici esortandolo a non occuparsi del loro senso e del loro 'codice': "lasciali digrignar pur a lor senno". Dante riusa lo schema del quarto e ottavo canto dell'Inferno per assegnare, in una sfumatura parodistica, un codice, un segnale convenzionale ed un maestro anche ad una schiera di diavoli, con la quale ancora una volta i due pellegrini percorrono un tratto del loro cammino (notevole l'occorrenza di un 'catalogo' quale quello dei versi 118-123 e notevole il giudizio del Camporesi [citato nel commento al verso di Umberto Bosco e Giovanni Reggio]: "la trombetta di Barbariccia si oppone in netta specularità negativa alle trombe dei cavalieri" [per simili implicazioni "parodistiche" degli strumenti musicali vedi anche l'episodio di Mastro Adamo per la contrapposizione tamburo-liuto $\left.{ }^{4}\right]$ ). Ed alla sensibilità di Charles Singleton non è sfuggito il fatto che anche in un simile contesto diabolico, e cioè da un punto di vista speculare ed opposto a quello del mondo del bene, c'è spazio, e forse con maggiore effetto comico, per una dimensione di 'complicità': "The grotesque gesture (one has only to imagine what snouts these demons have) amounts to a 'wink' of complicity and delight at the prospect of the adventure ahead, in which the devils are going to trick Virgil and Dante": 
“[...] non vedi tu ch'e" digrignan li denti

e con le ciglia ne minaccian duoli?".

Ed elli a me : "Non vo" che tu paventi;

lasciali digrignar pur a lor senno,

ch'e' fanno ciò per li lessi dolenti".

Per l'argine sinistro volta dienno;

ma prima avea ciascun la lingua stretta

coi denti, verso lor duca, per cenno;

ed elli avea del cul fatto trombetta.

La prima occorrenza della diade nclla cantica del Purgatorio è nella apostrofe di Dante a Firenze che si trova nel sesto canto (127-51) e segue l'invettiva contro l'Italia. È davvero interessante notare come Dante usi "cenno" quasi ad indicare il progresso civile e giuridico che Atene e Sparta realizzarono. Dante personifica le due città, visto che peraltro l'apostrofe a Firenze agevola e prepara questo trattamento del tema, ed immagina che esse annuiscano al "viver bene" che, data la tensione civile e politica che pervade il contesto, mi pare evidente sia la rappresentazione della giustizia, categoria quasi sovrapponibile al 'buon governo' nella Weltanschauung dantesca. Ecco che, se si dà anche al "cenno" di Atene e di Sparta un valore concreto di saluto o di manifestazione affettuosa di complicità, la sostanza del rapporto di tipo 'pedagogico' insita nella coppia di vocaboli, e cioè quella che ha a che fare con il livello psicagogico del miglioramento personale e collettivo, viene assolutamente preservata. Le due città si trovano in una "bella scola" capeggiata dalla personificazione del "vivere civile" e cioè dalla Giustizia e scambiano un "cenno" con essa e questo "cenno", che è in realtà inteso in senso grandemente meritorio, è detto "picciolo" solo per l'esigenza dell'ironia dantesca ("Or ti fa lieta, ché tu hai ben onde: / tu ricca, tu con pace e tu con senno! / S'io dico 'l ver, l'effetto nol nasconde. / Atene e Lacedemona, che fenno / l'antiche leggi e furon sì civili, / fecero al viver bene un picciol cenno / verso di te, che fai tanto sottili / provedimenti [...]").

Nella seconda occorrenza purgatoriale (Purg. 19.85-89) della coppia cennosenno (la sesta della serie che prenderemo in esame) assistiamo ad un diverso trattamento della polarità espressa dai due sostantivi o, se si vuole, ad un loro uso particolare e ristretto. Il "senno" d in questo caso una deliberazione soggettiva di Dante, una sua intenzione o volontà successiva ad un discernimento, come poi sarà nell'episodio del commiato da Virgilio in Purg. 27.138-42. Ma, in ogni caso, il 'codice' di questo senno risulta essere del tutto chiaro all'interlocutore (che qui e nel citato episodio del commiato è Virgilio, mentre in Par. 15.70-73 si avrà Beatrice) il quale poi con un "cenno" consente che esso si trasformi in una azione: "Poi ch'io potei di me fare a mio senno". L'episodio in questione è quello del dialogo con Papa Adriano $\mathrm{V}$ per rivolgere la parola al quale a Dante basta incrociare lo sguardo di Virgilio ("e volsi li occhi a li occhi 
al segnor mio") per ricevere un segnale di permesso ("ond"elli m 'assentì con lieto cenno"). Ma il maestro, la guida che è "mare" di tutta la conoscenza, e a questo punto ci sia consentito dirlo, di tutta la sensibilità di cui l'uomo è capace, ove non bastasse la sua straordinaria intelligenza delle cose umane avrebbe comunque - per comprendere ciò che il suo discepolo desidera o ciò che per lui è necessario - l'ausilio del "disio" che Dante lascia spesso trasparire ("ciò che chiedea la vista del disio").

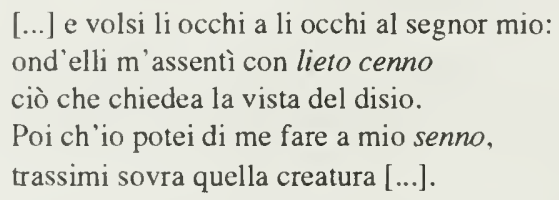

11 "disio" torna poi, come molla dell 'allocuzione, anche nel canto già citato del Paradiso (canto 15) e precisamente nelle parole di Cacciaguida che sa e prevede l'ansia che Dante ha di rivolgergli la parola ("la voce tua sicura, balda e lieta / suoni la volontà, suoni “l disio, / a che la mia risposta è già decreta!"). Mi preme sottolineare come nel "decreta" ci sia tutta l'ineluttabilità di quel volere divino che aveva già fatto incontrare Dante ed i poeti della "bella scola" ed aveva chiamato il pellegrino a quel viaggio che al suo principio egli aveva condiviso, anche se per poco, con la "schiera" dei grandi scrittori.

Nella settima occorrenza del sostantivo "cenno" che questa volta, e per ragioni come vedremo comprensibili, è orfano della controparte "senno", torniamo al significato generico che il vocabolo aveva per Dante e cioè non quello speciale di 'segno convenzionale' ma semp'icemente quello di 'cenno di saluto'. Si tratta del primo impatto con il poeta Stazio (Purg. 21.7-23), il primo incontro col personaggio che darà compimento alla temperie del Limbo. Il parallelismo e la contiguità del canto 4 dell'Inferno con il 21 del Purgatorio (incontro con Stazio) e con il 22 della stessa cantica (completamento del catalogo dei poeti) sono a mio giudizio schiaccianti ed ineludibili. I due pellegrini Dante e Virgilio procedono nel loro cammino ed improvvisamente l'“ombra" del poeta Stazio si avvicina a loro da dietro e li saluta, se non esattamente con un Pax vobis, con un "o frati miei, Dio vi dea pace" che s'accorda perfettamente col paragone che Dante ha appena fatto fra Cristo e Stazio: "Ed ecco, sì come ne scrive Luca / che Cristo apparve a' due ch'erano in via./ già surto fuor de la sepulcral buca, / ci apparve un'ombra, e dietro a noi venia". Anche i quattro grandi poeti del Limbo avevano salutato ed allo stesso tempo onorato Virgilio ("onorate l'altissimo poeta"); ma il loro 'saluto-encomio' resta, come tutto il mondo dei virtuosi pagani, al di qua del limite della Grazia, dalla quale Stazio è stato toccato proprio attraverso l'opera poetica di Virgilio. I quattro poeti avevano poi percorso un tratto di cammino con i pellegrini, così come anche Stazio si accinge a fare. Ma al conciliabolo tenuto dai cinque poeti (la 
"scola" con in più Virgilio) per decidere dell'ammissione di Dante nel loro "canone" ed al "cenno" di plauso e di lode per i meriti poetici del poeta e per i prodotti del suo "senno" non può corrispondere nulla di altrettanto compiaciuto o festoso nel contesto purgatoriale poiché quando Virgilio risponderà al saluto ("Noi ci volgemmo subiti, e Virgilio / rendéli "l cenno ch a ciò si conface") lo farà sottolineando l'importanza della meta della salvezza dalla quale egli è purtroppo escluso (" $[$... ] nel beato concilio / ti ponga in pace la verace corte / che me rilega nell 'etterno essilio"). Inutile sottolineare quanto amara è per Virgilio la differenza sottilmente adombrata tra "bella scola" e "beato concilio", tra i luoghi dove vive Omero, il "poeta sovrano" armato di spada e che incede "come sire", e la "verace corte" in cui egli non potrà mai entrare. La vasta sapienza di Virgilio non vale un posto in questo concilio e del suo "senno" non resta che una pallida traccia nell'appellativo "dottore" che Dante usa per lui ("E "l dottor mio: se tu riguardi a’segni [...]").

Una vera e propria riedizione del Limbo, o meglio della sua atmosfera spirituale, anche se in chiave di beatificazione, si trova invece al principio del canto 22 del Purgatorio (vv. 10-27); Virgilio, dopo aver cercato di contenere il prorompere dell affetto di Stazio (fine del canto 21) che, volendo abbracciare i piedi di Virgilio riporterebbe inutilmente le braccia al proprio petto (come Enea con Anchise) se il "Dottore" non gli ricordasse che, essendo entrambi delle ombre, questa dimensione fisica della gioia è loro preclusa, ripercorre con la memoria le tappe del suo rapporto personale con il poeta Stazio. Questo rapporto, cosa importantissima per noi, è poi squisitamente letterario in quanto per Stazio nasce dalla lettura delle opere di Virgilio (e specialmente, come Stazio stesso sostiene, della quarta Ecloga: "Ed elli a lui: "Tu prima m inviasti / verso Parnaso a ber ne le sue grotte, / e prima appresso Dio m alluminasti" [22.64-66]) mentre per Virgilio, acquisterà un senso profondo grazie all 'intermediazione del poeta Giovenale che, una volta disceso nella "casa com une che il Limbo è per i poeti. gli comunicherà l’amore del poeta tolosano (" [... G Giovenale / che la tua affezion mi fé palese" [22.14-15]). Come si vede gli elementi per un amor cortese nella sua famosa variante di 'amore di lontano' ci sono tutti ed evidentemente questa è l'unica dimensione possibile dell'incontro visto che Virgilio non pud ricevere da Stazio la opportunita di salvezza di cui invece questi gli è debitore. Ed è proprio per questo che, da una parte, Stazio. ringraziando esplicitamente Virgilio, tratterà il tema importantissimo di come egli ricevelte il dono della fede e di come egli ebbe quella che Sapegno nel commentare il luogo chiama "iniziazione alla poesia". mentre Virgilio stesso dall'altra onorerà lo spirito di Stazio rendendolo formalmente partecipe del "senno" del Limbo ed 'investendolo' con un particolare "cenno" che è qui il "dir d'amor". Credo infatti che nell "episodio siano contenuti almeno tre segnali che provano il fatto che Dante, in una stringente allusivita di tipo intratestuale, voglia esplicitamente 'citare' il quarto ed il quinto canto dell'Inferno in primo luogo per conferire formalmente a Stazio la qualifica di poeta degno del 
sommo 'canone' del quarto canto, in seconda istanza per mostrare come l'amore di tipo virtuoso (e per di più letterario!) abbia lo stesso potere di costringere a riamare così come lo ebbe l'amore di tipo peccaminoso o passionale di cui furono vittime Paolo e Francesca (e per giunta con la complicità di un libro!) e poi, ultimo elemento, per connotare quel "cenno" - che per Dante era stato di spinta ed esortazione alla salvezza ("salutevol"), del semplice e profondo sentimento d' amore di tipo cortese che si respira in tutta la Cantica ed in toni piuttosto simili a questo soprattutto negli episodi di Casella, Sordello, Guido Guinizelli ed Arnaldo Daniello (e sorprendenti sono i riscontri nel tipo di lessico usato per descrivere gli slanci d'affetto, gli abbracci 'possibili' [Sordello e Virgilio che si abbracciano a dispetto della loro condizione di ombre in una peculiare incongruenza della Commedia] ed 'impossibili' [Dante e Casella, così come Enea ed Anchise ed anche Virgilio e Stazio] che si verificano durante questi riconoscimenti tra musici, trovatori e poeti). Il primo segnale che ci troviamo innanzi è quello dell" amore di lontano": "[...] Amore, / acceso di virtu, sempre altro accese, / pur che la fiamma sua paresse fore;/onde de l'ora che tra noi discese / nel limbo de lo 'nferno Giovenale / che la tua affezion mi fé palese, / mia benvoglienza inverso te fu quale / più strinse mai di non vista persona [...]" con l"evidente variatio in imitando di "amor che a nullo amato amar perdona" e con l'uso del verbo "strinse" usato per Lancillotto, protagonista di quella lettura che ebbe il potere di perdere per sempre Paolo e Francesca. La seconda allusione è la più scoperta ed equivale ad un re-make dell'investitura poetica di Dante nel quarto dell'Inferno, ma questa volta senza conciliabolo di una 'commissione' di poeti 'canonici' che "ragionano alquanto" e nella forma di una fraterna ed amichevole curiosità di Virgilio circa la situazione di Stazio ed il motivo della sua espiazione: "Ma dimmi, e come amico mi perdona / se troppa sicurtà m'allarga il freno / e come amico omai meco ragiona: / come poté trovar dentro al tuo seno / loco avarizia, tra cotanto senno / di quanto per tua cura fosti pieno?". Virgilio dà quindi per scontata l'elevatezza d'ingegno e di sentimenti di quello che egli chiama per ben due volte amico ed addirittura, a proposito di questa scontata elevatezza di animo e di ingegno del poeta tolosano, manifesta il suo stupore sul fatto che poesia ed avarizia possano esser congiunte; così Dante consegue il risultato poetico di porre una enfasi ancora maggiore sulla inconciliabilità del difetto in questione con la padronanza dell'arte. Stazio, infatti, non espia l'avarizia bensì il suo contrario (e cioè la prodigalità) e le parole di Virgilio sono raffinatamente 'prodighe' di elogi per l'amico e di umiltà per i suoi stessi meriti, quasi a voler smentire, in un tale contesto 'cortese', che la sua opera sia stata determinante per la salvezza di Stazio ("di quanto per tua cura fosti pieno") e a voler sottolineare de facto la magnanimità dei poeti. Il terzo ed ultimo segnale è strettamente connesso al precedente e consiste nella trasformazione del "salutevol cenno" (rivolto dai quattro poeti del 'canone' a Dante, dopo che essi hanno tenuto concilio su di lui e sulle sue qualita), nella sorridente constatazione di 
Stazio che non pud non riconoscere nelle parole di Virgilio un "caro cenno" di "dir d'amore" e cioè il calore dell' amicizia e dell'amor cortese di cui si è detto. Egli, ugualmente, non può fare a meno di rendere soddisfazione all'amico quasi rispondendo ad un complice segno convenzionale ed accetta l'onore che Virgilio gli rende con l'uso del vocabolo cenno il quale invece che "salutevole" qui per lui può essere solamente "caro" nel senso più esclusivo e struggente che la malinconica situazione di Virgilio, ancorché sottintesa, può evocare: "Queste parole Stazio mover fenno / un poco a riso pria; poscia rispuose: / Ogne tuo dir d'amor m'è caro cenno".

L'ultima occorrenza della diade nel Purgatorio si ha significativamente negli ultimi quattro versi pronunciati da Virgilio all'indirizzo di Dante prima di scomparire (Purg. 27.139-142). L' 'iniziazione' del discepolo è ormai ratificata e conclusa. Le viene solamente conferita una solenne veste formale perché essa si trova a coincidere con un congedo. Dante, dopo la sosta successiva al superamento del muro di fuoco (è notevole come Virgilio, per rianimare Dante che è colto da grande timore, gli menzioni l'episodio di Gerione [versi 22-24 dello stesso canto 27] ed il suo esito felice, episodio in cui come si è visto la diade "senno"-"cenno" ha la sua massima connotazione nel senso del disvelamento di un arcano: "Ricorditi, ricorditi! E se io / sovresso Gerion ti guidai salvo,/ che farò ora presso più a Dio?") e necessaria per il riposo notturno, si risveglia. Al suo levarsi trova Virgilio e Stazio desti e pronti a salire sugli ultimi gradini della scala ed anche pronti a quello che Dante non sa ancora essere il commiato della sua guida ("[...] le tenebre fuggian da tutti lati / e 'l sonno mio con esse; ond'io leva'mi, / veggendo i gran maestri già levati" [112-14]: giustamente il Sapegno collega "gran maestri" a "gran marescalchi" di Purg. 24.99). A questo punto Virgilio ribadisce la vicinanza al conseguimento di quella felicita ("Quel dolce pome che per tanti rami / cercando va la cura de' mortali / oggi porrà in pace le tue fami" [115-17]) che è l'obiettivo di tutte le azioni umane ed in un certo senso rinsalda quell'aspettativa dell'incontro con Beatrice che già aveva instillato nell'animo di Dante proprio nei tribolatissimi istanti del passaggio attraverso il muro di fuoco ("[...] Or vedi figlio / tra Beatrice e te è questo muro" [35-36]). Il risultato della promessa di Virgilio è tonificante per l'anima di Dante ed esalta la forza del suo desiderio di ascesa e di purificazione. Il modo in cui Dante esprime questa esaltazione della volontà è straordinariamente vicino (se si studia l'uso che Dante fa delle due parole "ali" e "penne" nella Commedia si scopre che praticamente queste sono le uniche due occorrenze esattamente 'sovrapponibili' del poema: Purg. 27.121-23 e Par. 15.70-72) a quello con cui il poeta esprimerà il suo fortissimo desiderio di parlare con lo spirito che ignora esser quello del suo antenato Cacciaguida: Purg. 27.121-23: "Tanto voler sopra voler mi venne / de l'esser sù ch'ad ogne passo poi / al volo mi sentia crescer le penne"; Par. 15.70-72: "Io mi volsi a Beatrice e quella udio / pria ch'io parlassi, e arrisemi un cenno / che fece crescer l'ali al voler mio". Importante poi la ricorrenza del riso ("arrisemi") e della solita 'lettura del pen- 
siero" che questa volta in una fine variatio rispetto all'episodio di Gerione (di cui era stato protagonista Virgilio) è colta come un 'udire prima che si parli' ("udio pria ch"io parlassi"). Terminata l'ascesa e quindi proprio al "grado superno", allo stesso tempo simbolico e concreto, delle fatiche sostenute durante il viaggio oltremondano insieme al coadiutore che rappresenta la massima virtù umana possibile senza intervento della Grazia ("Come la scala tutta sotto noi / fu corsa e fummo in su' l grado superno / in me ficcò Virgilio li occhi suoi [...]" [124-26]) ha luogo finalmente l'ultima allocuzione di Virgilio a Dante. Nell'allocuzione [vv. 127-42] si tira mirabilmente il capo del filo che congiunge tutta la serie di 'investiture', 'complicità' e possibili 'riconoscimenti' o 'annuizioni' fra personaggi di pari dignità o comunque legati dal legame privilegiato maestro-discepolo che abbiamo incontrato fin qui. Dico mirabilmente perché a ben vedere l'allocuzione di Virgilio è un piccolo capolavoro di retorica ed allo stesso tempo di 'classicismo', almeno per ciò che riguarda la struttura perfettamente simmetrica delle frasi, il ritmo perfetto dei cinque periodi ciascuno dei quali occupa una ed una sola terzina (ad esclusione, ovviamente, dell'ultimo verso, il 142 che è sì isolato, ma contiene la formula definitiva della liberazione di Dante dalla cattiva volontà e dalla tentazione) e la funzionalità non disgiunta dallo stile delle allusioni a tutta la precedente porzione dell'opera. Virgilio parte dalla constatazione letterale dell'entità del cammino compiuto per poi toccare il tema più allegorico della sua propria impossibilità a continuare l'opera di guida nel regno della Grazia e si rivolge a Dante per la terza volta in questo canto $(20,35,128)$ con l'appellativo "figlio", in perfetta corrispondenza al "dolce padre" di verso 52 ("Lo dolce padre mio, per confortarmi. / pur di Beatrice ragionando andava, / dicendo "Li occhi suoi già veder parmi"). Ed il riferimento agli "occhi" ed alla "vista", portato avanti come vedremo anche nella quarta terzina, si fa vivo nel "discerno" e ci riporta alla catena Maria-Beatrice-Lucia (proteturice della vista, appunto e connessa all'idea della luce) ed allo stesso tempo ai versi d'addio e commiato con cui si chiudeva il quarto canto dell'Inferno ("La sesta compagnia in due si scema / per altra via mi mena il savio duca, / fuor de la queta, ne l'aura che trema. / E vegno in parte ove non è che luca"). Con in mente un ultimo richiamo al verbo "scemare" che sarà riusato da Dante nel momento in cui si accorgerà della scomparsa di Virgilio (Purg. 30.49-50: “[...] ma Virgilio n’avea lasciati scemi / di sé, Virgilio dolcissimo patre [...]”), leggiamo la prima terzina:

e disse "Il temporal foco e l'etterno veduto hai, figlio; e se' venuto in parte dov'io per me più oltre non discerno.

Nella seconda terzina Virgilio, in due cola perfettamente corrispondenti, che incastonano i due concetti ora non più antitetici "duce" $\mathrm{e}$ "piacere", riven- 
dica l'importanza dell' "ingegno" e dell " "arte" che hanno permesso a Dante di superare sentieri "erti” (ripidi) ed "arti" (impervi) prima di recuperare una retta volontà a cui piace ciò che è giusto:

Tratto t'ho qui con ingegno e con arte;

lo tuo piacere omai prendi per duce:

fuor se'de l'erte vie, fuor se' de l'arte.

La terza terzina, quasi in una rievocazione-citazione della lucentezza di Catone, allude al sole della Grazia che risplende sul volto di Dante e riproduce, ma questa volta in un atmosfera di grande speranza e non di disperazione, il locus amoenus che c'era nel Limbo dei pagani virtuosi e che qui si invera nella pienezza dell'Eden (da notare ancora la perfetta simmetria di "sol" e "sol" attorno al perno del verso centrale come nella terzina precedente i due cola opportunamente evidenziati):

Vedi lo sol che 'n fronte ti riluce; vedi l'erbette, i fiori e li arbuscelli che qui la terra sol da sé produce

Nella quarta, nitida terzina c'è il ritorno al tema stilnovistico degli occhi di Beatrice, colti però nel loro esortare Virgilio il Venerdì Santo, quando avvenne la discesa al Limbo della donna-angelo, e a Dante è formalmente accordata la possibilità di riposare sul prato nell'attesa della 'epifania' di Beatrice (notevole il doppio uso del chiasmo per "venire", che incastona gli epiteti degli occhi, e per "puoi" che, al contrario, e incastonato fra i due verbi che esprimono il formale permesso del riposo):

Mentre che vegnan lieli li occhi belli che, lagrimando, a te venir mi fenno, seder ti puoi e puoi andar tra elli .

L'ultima terzina, col suo verso-coda che sancisce l" "espiazione-iniziazione' del pellegrino ("per ch'io te sovra te corono e mitrio": qui c'è quella che potrebbe esser considerata una 'dittologia sinonimica' che però si arricchisce di sfumature, in relazione ai due 'poteri' ed ai due tipi di 'corte' che i verbi "corono" e "mitrio" evocano - se soltanto pensiamo ai connotati cavallereschi del rapporto fra poeti che abbiamo rintracciato ed al culminare del tema dell' 'esame' nel colloquio con San Pietro), svincola Dante da ogni valutazione del suo arbitrio che, essendo giudicato retto, sano e libero, è per cio stesso in possesso se non immediatamente della verità almeno del "senno" necessario per interpretarne il 'codice'. Dante è quindi pronto; virtualmente egli ha superato addirittura i limiti dell'appartenenza alla "bella scola": 
"Non aspettar mio dir più né mio cenno;

libero, dritto e sano è tuo arbitrio,

e fallo fora non fare a suo senno:

per ch'io te sovra te corono e mitrio".

Veniamo quindi all "ultima occorrenza della coppia "cenno"-"senno" che è appunto quella del canto di Cacciaguida (Par. 15.25-75). È opportuno ricordare ciò che Cacciaguida rappresenta. Egli è l'avo di Dante e questo porta al ricongiungimento del poeta con il "suo stesso sangue" come fu per Enea nell'incontro con Anchise che è direttamente citato dal poeta ("[...] sì pia l'ombra d'Anchise si porse, / se fede merta nostra maggior musa, / quando in Eliso del figlio s'accorse"). Questo fatto, a sua volta, permette di estendere l'aura della presenza virgiliana anche a questo importantissimo punto del poema. Cacciaguida rappresenta allo stesso tempo la nobiltà di sangue e l'essenza profonda dell'appartenenza al mondo cavalleresco nel senso più umanamente dignitoso perché egli, prima di parlare di sé come cavaliere di Corrado III di Svevia in Terra Santa, rimprovera le manifestazioni esteriori del lusso e della corruzione dei costumi fiorentini; un rimprovero che culmina nel brevissimo chiasmo-catalogo di esempi negativi del presente contrapposti a quelli, neanche a dirlo, positivi della romanità: "Saria tenuta allor tal maraviglia / una Cianghella, un Lapo Salterello, / qual or saria Cincinnato e Corniglia. / A cosi riposato, a cosi bello / viver di cittadini, a così fida / cittadinanza, a così dolce ostello, / Maria mi diè, chiamata in alte grida; / e ne l'antico vostro Batisteo / insieme fui cristiano e Cacciaguida. / Moronto fu mio frate ed Eliseo; / mia donna venne a me di val di Pado, / e quindi il sopranome tuo si feo./Poi seguitai lo 'mperador Currado; / ed el mi cinse della sua milizia, / tanto per bene ovrar li venni in grado " (127-41). Notevole poi come anche qui, nell'elogio di Firenze, che riecheggia, riprende e completa l'invettiva di Purg. 6, il "viver bene" diventi "riposato" e "bello" ma resti pur sempre una personificazione a cui la Vergine consegna il pargoletto (ed a cui invece Atene e Sparta avevano solo annuito). Ho detto che questo è un punto importantissimo del poema proprio perché Dante riceverà da Cacciaguida un nuovo tipo di 'investitura' e quindi nuovi ardui compiti; credo che la onniscenza e cioè, oltre alla normale dottrina, anche la conoscenza del futuro dovuta alla beatitudine - tratto che collega i Beati a Dio stesso - sia la chiave della parola "senno" nella sua ultima occorrenza che si trova appunto in questo canto. I compiti assegnatigli da Caccaguida sono inoltre tutti proiettati alla fine del viaggio ultramondano e riguardano la vita futura di Dante e, pertanto, non sono riconoscimenti dal valore retroattivo o istantaneo come la cooptazione nella "bella scola" o la liberazione dalla schiavitù del peccato ad opera della 'corona' e della 'mitra'di Virgilio. Il primo compito è ovviamente quello della preghiera e delle buone azioni per agevolare l'espiazione del figlio Alighiero: "Poscia mi disse: Quel da cui si dice / tua cognazione e che cent'anni e piùe / girato ha '1 monte in la 
prima comice, / mio figlio fu e tuo bisavol fue: / ben si convien che la lunga fatica / tu li raccorci con l'opere tue" [91-96]. Il secondo, ma verrà chiaramente enunciato dall'antenato solo nel canto 17 del Paradiso, è conseguenza proprio di ciò che fa ardere Dante di desiderio (Par. 17.1-12 con la solita 'lettura del pensiero' operata da Beatrice e da Cacciaguida) ed è cioè la risposta agli interrogativi sul suo futuro. Cacciaguida gli predice l'esilio e la necessità di accettarlo con rassegnazione e senza odio per i suoi concittadini.

È a questo livello di conoscenza delle cose arcane che Dante si appellerà spinto come sempre a manifestare la sua curiosità da Beatrice (siamo ancora nel canto 17 del Paradiso [13-27]) - e, cosa estremamente significativa, nel farlo citerà il nome di Virgilio, prima guida e primo maestro da cui egli fu messo al principio dentro le "segrete cose" e da cui fu accompagnato nel viaggio oltremondano così pieno di oscuri presagi intorno al suo futuro:
"O cara piota mia che sì t'insusi, che come veggion le terrene menti non capere in triangol due ottusi, così vedi le cose contingenti anzi che sieno in sé, mirando il punto a cui tutti li tempi son presenti; mentre ch'io era a Virgilio congiunto su per lo monte che l'anime cura e discendendo nel mondo defunto, dette mi fuor di mia vita futura parole gravi, avvegna ch'io mi senta ben tetragono ai colpi di ventura. Per che la voglia mia saría contenta d'intender qual fortuna mi s'appressa; ché saetta previsa vien più lenta".

E così, tornando al primo impatto, al primo incontro con lo spirito di Cacciaguida (canto 15), credo si possa concludere la nostra analisi della diade "senno"-"cenno" notando come tutto l'insieme narratologico dei fatti che si accompagnano al tema dell' annuizione, della complice investitura, del maestro che 'capisce al volo' i dubbi e le curiosità di un discepolo e li scioglie e le soddisfa, sia anche in questo episodio del tutto rispettato.

Appena Cacciaguida si manifesta, citando, per così dire, Virgilio e parlando in latino, Beatrice sorride con la sicurezza che sempre accompagna le guide: "[...] poscia rivolsi a la mia donna il viso, / e quinci e quindi stupefatto fui; / ché dentro a li occhi suoi ardeva un riso / tal, ch'io pensai co' miei toccar lo fondo / de la mia gloria e del mio Paradiso" [32-36].

Quando Cacciaguida riprende la parola non manca di benedire Dio e di qualificare Dante con l'appellativo "cortese" riportandoci a quella solennità e serietà con cui Virgilio l'aveva 'mitriato' e 'coronato' e con cui i poeti della 
"scola" avevano salutato il ritorno del loro compagno Virgilio: "la prima cosa che per me s'intese, / 'Benedetto sia tu', fu, 'trino e uno, / che nel mio seme se' tanto cortese!'" [46-48].

Nella continuazione Cacciaguida lo chiama "figlio" e menziona poi Beatrice come colei che gli donò le "piume" per 1"“alto volo": "[...]"Grato e lontano digiuno, / [...] solvuto hai, figlio, dentro a questo lume / in ch'io ti parlo, mercé di colei / ch' a l'alto volo ti vestí le piume'." [49-54].

L'avo di Dante lascia poi intuire i suoi poteri, comuni peraltro a tutti i beati, di leggere il pensiero e rincuora Dante incoraggiandolo a formulare le sue domande (55-69) a cui la risposta di Cacciaguida è "decreta", come abbiamo già detto, per volere divino.

Dante è quindi pronto a ricevere un "cenno" dalla sorridente Beatrice e ad alludere alla superiorità del "senno" che Cacciaguida rappresenta rivolgendosi a lui, di cui non conosce il nome, proprio apostrofandolo come partecipe di quell'amore e di quella superiore sapienza da cui egli si accinge a ricevere le direttive per il resto della vita che lo attende alla fine del viaggio. Si tratta dunque dell'ultimo grande viatico e dell'ultimo segreto da svelare, un segreto di cui il 'codice' è per definizione inconoscibile. Ė come se Cacciaguida, quale consanguineo, accogliesse Dante presso di sé come un 'figliol prodigo', visto anche che il Poeta suggerisce l'immagine ringraziando l'avo per "la paterna festa" [70-87]:

Io mi volsi a Beatrice, e quella udio pria ch'io parlassi, e arrisemi un cenno che fece crescer l' ali al voler mio. Poi cominciai così: 'l'affetto e 'l senno, come la prima equalità v'apparse, d'un peso per ciascun di voi si fenno però che 'l sol che v'allumò e arse, col caldo e con la luce è sì iguali, che tutte simiglianze sono scarse . Ma voglia e argomento ne' mortali, per la cagion ch'a voi è manifesta, diversamente son pennuti in ali; ond'io, che son mortal, mi sento in questa disagguaglianza e però non ringrazio se non col core alla paterna festa. Ben supplico io a te, vivo topazio che questa gioia preziosa ingemmi, perché mi facci del tuo nome sazio'.

Voglio da ultimo far notare la continua ricorrenza dell'illuminazione da parte del sole che qui chiude la serie 'incontro con Catone-commiato da Virgilioincontro con Cacciaguida'. 
Il tema dell'esame e dell'investitura sarà ripreso, completato e portato alle sue estreme conseguenze, senza che appaia più la coppia "cenno"-"senno", ma in modo che ne sopravviva l"intero bagaglio di motivi e suggestioni che siamo venuti fin qui rintracciando, nell' episodio in cui il "barone" S. Pietro esamina e "tenta" sulla fede il "baccialliere" Dante.

Grazie a questo episodio, che si trova in Par. 24, Dante può davvero superare la "bella scola", la sua eredità e la sua stessa appartenenza ad essa. Egli, infatti. come il tema adombrato nel canto di Cacciaguida lasciava presagire, pone in una urgente attualità il rito dell'esame e dell'investitura e, mentre da una parte usa uno schiacciante riferimento al mondo universitario a lui contemporaneo (il paragone col "baccialliere" che difende una tesi di fronte ad un "maestro" dei versi 46-51), pone dall'altra come obiettivo ultimo del suo poetare (ed in ultima analisi della poesia in generale come arte) quello della promozione spirituale del genere umano e cioè proprio quel livello psicagogico a cui abbiamo spesso alluso. L'esame chiude il canto 24 e subito all'apertura del 25 vi è la chiara proclamazione del desiderio di Dante di essere incoronato poeta, inquantoché egli si sente a ciò legittimato dalla positiva valutazione di San Pietro (e non certo di quella espressa dalla "bella scola"!), e proprio nella sua patria: siamo lontanissimi dall'atmosfera 'marginale' di esilio dalla storia (cioè quella successiva alla venuta di Cristo, quella che sola conta) e dalla Grazia che era propria degli abitanti del Limbo. Dante, per giunta, spera di superare, di vincere proprio il suo personale 'esilio' anche grazie al suo poema (1-12):

Se mai continga che "l poema sacro al quale ha posto mano e cielo e terra, sì che $m$ 'ha fatto per molti anni macro, vinca la crudeltà che fuor mi serra del bello ovile ov'io dormi' agnello, nimico ai lupi che li danno guerra; con altra voce omai, con altro vello ritornerò poeta, $e$ in sul fonte del mio battesmo prenderò ' 1 cappello; però che ne la fede, che fa conte l'anime a Dio, quivi intra'io, e poi Pietro per lei sì mi girò la fronte.

Su questa ultima tappa che dà il significato stesso del tema della investiturainiziazione nel poema, nel viaggio del pellegrino Dante non solo attraverso i regni dell'oltretomba ma anche attraverso differenti gradi di sapere e differenti gradi di consapevolezza di questo sapere (Virgilio ed Omero non hanno alcun titolo per 'consacrare' ma possono soltanto investire un collega più fortunato dal punto di vista dei temi psicagogici della sua poesia!), non posso non citare il magnifico commento al passo di Natalino Sapegno, che, a sua volta, cita G. Getto: 
Il canto [25] si apre con un movimento lirico, che è tra le note più umane di tutto il Poema, e al tempo stesso suggella il significato rituale di tutto l'episodio. Il rito dell'esame si trasforma in una cerimonia di consacrazione. Le note personali [...] si fondono con il tema di una missione universale. "L'incoronazione di Dante" significa anche "il riconoscimento della validità del suo messaggio".

Ma facciamo un passo indietro proprio per vedere meglio l'episodio dell'esame di cui abbiamo anticipato le conclusioni e le implicazioni ( $P a r$. 24.34-51). Anche in questo caso ritengo mirabile il giudizio di Sapegno in nota al verso 50, proprio perché coglie, forse in misura maggiore rispetto agli altri commentatori, il legame della 'situazione esame' con la ritualità e quindi in un certo modo avalla il nostro accostamento dell'episodio con il quarto canto dell'Inferno e con la rituale cooptazione di Dante nella "bella scola", al di là ovviamente dello scontato collegamento esame-baccelliere-scuola:

Il tono alto dell'apostrofe di Beatrice ai santi e del colloquio fra Beatrice e Pietro, e quella e questo tramati di immagini scitturali ed improntati a un decoro di cerimonia liturgica; l'immagine stessa del baccelliere, che insiste sull'atteggiamento di concentrata e tesa aspettazione di Dante e sull'importanza dell' atto a cui egli s'accinge e l'autorità di coloro che son chiamati a giudicarlo; costituiscono un solenne preludio all'episodio e ne determinano il carattere rituale e di solenne consacrazione e di suprema conferma alla missione morale e religiosa del poeta.

Beatrice invita San Pietro, chiamato "gran viro", a saggiare le conoscenze di Dante sulla fede (34-45):

Ed ella: "O luce etterna del gran viro a cui Nostro Signor lasciò le chiavi, ch' ei porlò giù, di questo gaudio miro, tenta costui di punti lievi e gravi, come ti piace, intorno de la fede, per la qual tu su per lo mare andavi.

S'elli ama bene e bene spera e crede, non l'è occulto perché 'l viso hai quivi dov'ogne cosa dipinla si vede; ma perché questo regno ha fatto civi per la verace fede, a gloriarla, di lei parlare è ben ch'a lui arrivi."

Non resisto alla tentazione di amplificare il senso di quel "portar giù" nella direzione del Descensus Christi ad Inferos per la liberazione dei Patriarchi, di legare "regno" e "civi", al di là dell' immediata cifra evangelica, al 'regno' del nobile castello con i suoi sfortunati "viri" non liberati da Cristo come furono i Patriarchi (che infatti condividono il "gaudio miro") e di ribadire l'inveramento che l'esame di San Pietro costituisce per Dante, sottolineando come agli "dei falsi e bugiardi" si contrapponga quella che già lo stesso Virgilio (rivol- 
gendosi a Stazio) aveva chiamato la "verace corte" dove c'é posto solamente per la "verace fede". Ho messo in risalto, inoltre, le parole di Beatrice che ripropongono il solito motivo della lettura della mente altrui comune a tutti i beati.

Quindi Dante instaura il paragone con lo studente universitario (non a caso delle scuole teologiche!) e definisce formalmente San Pietro "maestro" (un povero pescatore trasformato dal mistero della predestinazione in principe degli Apostoli, come lo stesso Dante dirà nei versi 109-110: “[...] ché tu intrasti povero e digiuno / in campo, a seminar la buona pianta [...]"):

\section{Sì come il bacciallier s'arma e non parla fin che "l maestro la question propone, per approvarla, non per terminarla, così m'armava io d'ogne ragione mentre ch'ella dicea, per esser presto a tal querente e a tal professione. (46-51)}

Sopravvive peraltro anche il tema della complicità, quando Dante, prima di parlare, sebbene gliene abbia fatto richiesta San Pietro, che rappresenta di certo un grado di beatitudine più elevato di Beatrice o quanto meno una dignità funzionale più alta, chiede quasi permesso con lo sguardo alla "sua donna":

poi mi volsi a Beatrice, ed essa pronte sembianze femmi perch'io spandessi

l'acqua di fuor del mio interno fonte. (55-57)

Credo che qui la coppia "cenno-senno" (che sarebbe stata perfettamente calzante) non venga usata per l'importante ragione che l' 'iniziando' è ben al di dentro del "codice', possiede cioè un "senno" che deve essere solo mostrato e non formato, cesellato o comunque riconosciuto da altri colleghi.

E se imitazione di un re nel Limbo era stato Omero ("come sire" e non già "sire", "poeta sovrano" e non già "sovrano"), qui nel Paradiso di Cristo San Pietro e come lui San Giacomo (nel successivo canto 25 del Paradiso appena dopo i versi già citati sull'incoronazione poetica) ed anche Ugo il Grande (Par. 16.127-30) sono senza esitazione definiti "baroni", nelle uniche tre occorrenze del poema, quasi ad insistere sul tema della necessita di una ratifica morale dei titoli nobiliari:

Finito questo, l' alta corle santa risonò per le spere un "Dio laudamo" ne la melode che là su si canta.

$E$ quel baron che sì di ramo in ramo, essaminando, già tratto m'avea, che a l'ultime fronde appressavamo, ricominciò [...] (112-18) 
Dante ha così congiunto e fuso l'aspetto scolastico-universitario e quello epico-cavalleresco, che erano già in nuce nella cooptazione del pellegrino nella "bella scola" dei poeti del canone del canto 4 dell'Inferno, e che qui vengono così ampiamente ripresi ed esaustivamente sviluppati. Così anche negli ultimi versi che chiudono il canto credo che il trattamento 'feudale' del dialogo fra Dante e San Pietro, che qui è visto come un rendiconto patrimoniale tra un servo-amministratore ed un padrone, al di là dei facili riferimenti ai tanti servi ed alle tante vigne che nella Bibbia si trovano, valga come seria indicazione del livello 'cavalleresco' e 'nobiliare' che punteggia la Commedia sin dall 'incontro del "nobile castello":

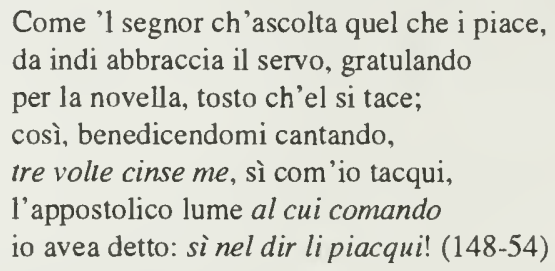

Voglio anche far notare come l'esser cinto tre volte dall'"apostolico lume" ricalchi in pieno, nella martellante insistenza sui temi 'cavalleresco e sacro', l'investitura a cavaliere di Cacciaguida ( $P$ ar. 15.139-44). Corrado III ordina cavaliere l'antenato di Dante "per bene ovrar" e quindi il poeta torna ad enfatizzare l'importanza della nobiltà solo in quanto frutto di meriti personali che, se si leggono simultaneamente i due episodi, possono essere guerreschi o letterari ("sì nel dir li piacqui!"), a patto che la letteratura abbia una forza 'psicagogica', generi cioè un 'poema sacro' che, per la coscienza individuale e collettiva, abbia la stessa forza liberatoria e lo stesso impatto guerresco della Crociata a cui partecipò Cacciaguida.

\section{Poi seguitai lo 'mperador Currado; ed el mi cinse de la sua milizia, tanlo per bene ovrar li venni in grado.}

Forzare una realtà di stile o di estetica in categorie prestabilite non è nostra intenzione, ma è pur chiaro che non ci si può sottrarre al richiamo evidente dei fatti.

Mi pare che una stringente strategia di tipo allusivo intratestuale che colleghi fra loro i luoghi citati sia a questo punto un fatto dimostrato. Fin qui niente di nuovo sotto il sole, poiché una strategia allusiva non è affatto strana in una BuchKultur. Ma ciò che mi interessa sottolineare è che questa allusività intratestuale dantesca deve fare i conti anche con la sostanza della poesia stessa, con il tipo di medium che viene impiegato. Voglio dire cioè che non tutte le connotazioni stilistiche di cui abbiamo parlato possono essere collocate sullo stesso 
piano gerarchico. Ciò che infatti ha colpito chi scrive, è che le parole-chiave della nostra ricerca sono poste in rima di terzina, e ciod sono in una posizione privilegiata del verso, così come (in compagnia di quella iniziale) quella finale era la posizione privilegiata nell'esametro greco e in quello latino. I due termini "cenno" e "senno" si imprimono benc nella memoria e quello che ci si chiede a questo punto è se riescano nello scopo soltanto per un puro fatto visivo. La terzina dantesca è una unità stilistica generalmente molto indipendente e crediamo quindi che la coppia di termini che in essa si incastona determinando la rima possa aver facilmente giocato anche un ruolo diverso da quello puramente visivo. Alludo ad una dimensione di 'oralità' che molti stilemi danteschi ed anche alcuni versi perché ripetuti più volte o particolarmente espressivi da un punto di vista acustico ci porterebbero ad ipotizzare per la Commedia così come è stato fatto per i poemi omerici (basti pensare al "vuolsi così colà dove si puote" della prima cantica o ai versi come "papè satan papè satan aleppe"). Cì che si è detto è da prendere cum grano salis nel senso che a nostro giudizio i due tipi possibili di fruizione convivono pacificamente nella poesia dantesca che puo essere orale (anche se non nel senso rigoroso in cui Ong parla a proposito dell'Antico Testamento o Havelock di Omero) ed anche 'chirografica' o 'tipografica' - come direbbe Marshall McLuhan - apprezzabile cioè appieno solo con il testo sotto gli occhi. Importanti appoggi per questa nostra supposizione sono le letture pubbliche (e senza dubbio anche private, specialmente durante la sua composizione) a cui il lavoro fu presto destinato, la particolare struttura chiusa e 'concatenata' della terzina dantesca, che è un grande aiuto per la memoria, e, da ultimo, ma di non poco momento, la possibilità di una allusione di tipo intratestuale che presuppone di necessità la capacità di coinvolgere la memoria del lettore. ${ }^{5}$ Questa memoria giocherà ovviamente sul macrolivello di importanti spie semantiche quali sono le parole "cenno" e "senno" di cui ci siamo occupati e che sono in rima di terzina e non agira invece in modo altrettanto fulmineo per altro tipo di dettagli che porteranno invece ad apprezzare l'arte dantesca solo dopo una lettura più minuziosa svolta con il libro davanti agli occhi (ecco perché ho parlato di 'gerarchie"?).

Ciò che ci lascia abbastanza fiduciosi nell'indicare in questa direzione un fertile campo di indagine è che la tecnica dantesca di cui si è discusso e che, come si è visto, coinvolge luoghi del poema fra loro strutturalmente collegati, è da rintracciare anche per altre coppie di vocaboli in rima di terzina, quali "alimortali", "spalle-calle", "spalle-valle", "lasso-passo", "grazia-sazia" e "frontemonte". Nell' attesa di dare ai dati una veste appropriata non resta che ribadire le conclusioni a cui Gian Biagio Conte ci ha abituati dall 'uscita del suo Memoria dei poeti: l'arte allusiva non ha limitazione di generi e di epoche e nel nostro caso essa potrebbe essere determinante per scoprire non solo come Dante 'lavorasse', ma anche come 'funzionasse' la sua memoria. 
NOTE

1 Per un inquadramento generale si rimanda al saggio introduttivo di lannucci in Dante e la "bella scola".

2 Un'analisi semantica consimile è quella di Bosco.

3 È qualcosa di cui Segre è consapevole quando scrive: "La Divina Commedia sta al culmine di altri due tipi di testi: il viaggio nell'altro mondo [...] e la visione dell' altro mondo [...]. Ma in questi due generi inietta anche elementi del viaggio allegorico. Del viaggio allegorico è la progressione degli insegnamenti, la presenza delle personificazioni, l'obbligo di esami per l'ammissione a livelli superiori di studio" (59).

4 Si veda l'esauriente contributo di lannucci "Musical Imagery".

5 Ottima la trattazione di Ahern.

\section{OPERE CITATE}

Ahem, John. "Singing the Book: Orality in the Reception of Dante's Comedy." Dante: Contemporary 214-239.

Alighieri, Dante. La Commedia secondo l'antica vulgata. Ed. Giorgio Petrocchi. 4 voll. Milano: Mondadori, 1966-67.

- La Divina Commedia. Ed. Natalino Sapegno. 3 voll. Firenze: La Nuova Italia Editrice, 1983.

-The Divine Comedy. Trad. e commento a cura di Charles S. Singleton. 3 voll. Princeton: Princeton UP, 1970-75.

Bosco, Umberto. "La 'follia' di Dante". Lettere Italiane 10 (1958): 417-30.

Bosco, Umberto, e Giovanni Reggio, eds. Dante Alighieri. La Divina Commedia. Firenze: Le Monnier, 1979.

Contellazzo, Manlio, e Paolo Zolli. Dizionario Etimologico della Lingua Italiana. Bologna: Zanichelli, 1988 (DELI).

Conte, Gian Biagio. Memoria dei poeti e sistema letterario: Catullo, Virgilio, Ovidio, Lucano. Torino: Einaudi, 1974.

Da una riva e dall' altra. Studi in onore di Antonio D'Andrea. Ed. Dante Della Terza. Firenze: Cadmo, 1995.

Dante: Contemporary Perspectives. Ed. Amilcare A. Iannucci. Toronto: U of Toronto P, 1997.

Dante e la "bella scola" della poesia: autorità e sfida poetica. Ed. Amilcare A. Iannucci. Ravenna: Longo, 1993.

Dizionario Etimologico Italiano. Ed. Carlo Battisti e Giovanni Alessio. Firenze: Barbera, 1968 (DEI).

Enciclopedia Dantesca. Ed. Umberto Bosco. 6 voll. Roma: Istituto della Enciclopedia Italiana, 1970-78.

Grande Dizionario della Lingua Italiana. Ed. Salvatore Battaglia. Torino: UTET, 1962 (GDLI).

Havelock, Eric A. Cultura orale e civiltà della scrittura. Da Omero a Platone. Bari: Laterza. 1983. Iannucci, Amilcare. "Musical Imagery in the Mastro Adamo Episode." Da una riva 103-18.

Niccoli, Alessandro. "Senno". Enciclopedia Dantesca. 5. 161-62.

Ong, Walter J. Oralità e scrittura. Le tecnologie della parola. Trad. di Alessandra Calanchi. Bologna: II Mulino, 1986.

Pasquini, Emilio. "Cenno". Enciclopedia Dantesca 1.908-9.

Segre, Cesare. Fuori del mondo: i modelli nella follia e nelle immagini dell' aldila. Torino: Einaudi, 1990.

Thesaurus Linguae Latinae. 9 voll. Leipzig: Teubner, 1900-1995.

Wahrig, Gerhard. Wörterbuch der deutschen Sprache. München: DTV, 1978. 